\title{
pks63787, a polyketide synthase gene responsible for the biosynthesis of benzenoids in the medicinal mushroom Antrodia cinnamomea
}

Po-Wei Yu, ${ }^{\dagger}$ Ya-Chih Chang, ${ }^{\S}$ Ruey-Fen Liou, ${ }^{\dagger}$ Tzong-Huei Lee, ${ }^{*}{ }^{\dagger}$ and Shean-Shong Tzean $^{*}{ }^{\dagger}$

${ }^{\dagger}$ Department of Plant Pathology and Microbiology, National Taiwan University, Taipei,

Taiwan 106; ${ }^{\S}$ College of Pharmacy, Taipei Medical University, Taipei, Taiwan 110; ${ }^{\star}$ Institute of Fisheries Science, National Taiwan University, Taipei, Taiwan 106 
Table S1. Quantity of extracts from A. cinnamomea wild type f101, transformant 78711 and 78714

\begin{tabular}{lccc}
\hline Extract solvent & $\mathrm{f} 101(\mathrm{wt})$ & 78711 (ectopic) & \multicolumn{1}{c}{$78714(\Delta p k s 63787)$} \\
\hline Ethyl acetate extract & $26.5 \pm 0.7$ & $26.1 \pm 0.1$ & $16.2 \pm 0.6^{*}$ \\
$n$-Hexane extract & $13.7 \pm 0.4$ & $13.4 \pm 0.2$ & $6.7 \pm 0.4^{*}$ \\
\hline
\end{tabular}

Each sample incubated on PDA for 30 days. Values shown represent as the mean \pm SD (mg/plate).

* Indicates a significant difference from the wild type group $(p<0.01)$ 
Table S2. NMR ${ }^{1} \mathrm{H}$ Spectroscopic Data $(500 \mathrm{MHz})$ for compounds 1-7 [ $\delta$ in ppm, mult. $(J$ in $\left.\mathrm{Hz})\right]$

\begin{tabular}{|c|c|c|c|c|c|c|c|}
\hline position & $\mathbf{1}^{a}$ & $2^{a}$ & $\mathbf{3}^{a}$ & $4^{b}$ & $\mathbf{5}^{b}$ & $6^{b}$ & $7^{b}$ \\
\hline \multicolumn{8}{|l|}{1} \\
\hline 2 & & & $5.95, \mathrm{~d}(2.4)$ & & $6.41, \mathrm{~s}$ & & \\
\hline \multicolumn{8}{|l|}{3} \\
\hline 4 & & & $6.54, \mathrm{~m}$ & $6.40, \mathrm{q}(1,6)$ & & & $6.28, \mathrm{~s}$ \\
\hline 5 & $6.34, q(0.7)$ & $6.40, \mathrm{~s}$ & & & & $6.41, \mathrm{~s}$ & \\
\hline \multicolumn{8}{|l|}{6} \\
\hline 7 & $2.08, \mathrm{~d}(0.6)$ & $2.10, \mathrm{~s}$ & 2.01, d (1.6) & $2.01, \mathrm{~d}(1.6)$ & $2.18, \mathrm{~s}$ & $2.19, \mathrm{~s}$ & $2.15, \mathrm{~s}$ \\
\hline 8 & $3.81, \mathrm{~s}$ & $3.78, \mathrm{~s}$ & $3.81, \mathrm{~s}$ & $3.99, \mathrm{~s}$ & $3.76, \mathrm{~s}$ & $3.78, \mathrm{~s}$ & $3.82, \mathrm{~s}$ \\
\hline 9 & $3.80, \mathrm{~s}$ & $3.70, \mathrm{~s}$ & & $3.96, \mathrm{~s}$ & $3.91, \mathrm{~s}$ & $3.47, \mathrm{~s}$ & $5.90, \mathrm{~s}$ \\
\hline 10 & & & & & $3.82, \mathrm{~s}$ & $3.93, \mathrm{~s}$ & $3.85, \mathrm{~s}$ \\
\hline
\end{tabular}

${ }^{a}$ Measured in methanol- $d_{4} ;{ }^{b}$ Measured in chloroform- $d$ 
Table S3. NMR ${ }^{13} \mathrm{C}$ Spectroscopic Data (125 MHz) for compounds 1-7 [ $\delta$ in ppm]

\begin{tabular}{|c|c|c|c|c|c|c|c|}
\hline position & $\mathbf{1}^{a}$ & $2^{a}$ & $\mathbf{3}^{a}$ & $4^{b}$ & $\mathbf{5}^{b}$ & $6^{b}$ & $7^{b}$ \\
\hline 1 & 142.1 & 143.2 & 160.7 & 145.0 & 137.0 & 141.1 & 138.6 \\
\hline 2 & 142.3 & 137.2 & 108.0 & 144.8 & 107.9 & 139.9 & 134.7 \\
\hline 3 & 140.2 & 138.7 & 189.4 & 184.2 & 121.1 & 146.0 & 136.5 \\
\hline 4 & 143.6 & 142.3 & 134.5 & 131.2 & 145.3 & 140.0 & 108.8 \\
\hline 5 & 113.2 & 110.5 & 145.3 & 144.0 & 140.5 & 109.5 & 123.6 \\
\hline 6 & 121.1 & 115.2 & 183.5 & 184.4 & 143.2 & 117.9 & 138.8 \\
\hline 7 & 15.8 & 15.6 & 15.3 & 15.4 & 15.5 & 15.5 & 15.9 \\
\hline 8 & 61.1 & 60.7 & 56.9 & 61.2 & 56.3 & 56.6 & 59.9 \\
\hline 9 & 61.2 & 57.3 & & 61.1 & 60.8 & 60.9 & 101.4 \\
\hline 10 & & & & & 60.5 & 61.2 & 59.9 \\
\hline
\end{tabular}

${ }^{a}$ Measured in methanol- $d_{4} ;{ }^{b}$ Measured in chloroform- $d$ 
Table S4. List of primers designed in present study

\begin{tabular}{|c|c|c|}
\hline Primer name & Primer sequence $\left(5^{\prime}-3^{\prime}\right)$ & Remarks \\
\hline 63787-cDNA-f & CAACTAGCCGTTCAATTCAGACG & pks63787 cDNA cloning \\
\hline 63787-cDNA-r & GAAGGGCCCACCGTCTCTA & pks63787 cDNA cloning \\
\hline 63787-3'-race & CGTTCTCATCGATTCTCCGCACCCG & 3' RACE \\
\hline 63787-5'-race & CATTGGGAACCGAACATGTCGTCTTGC & 5' RACE \\
\hline KS-p1 & TGCGACTGGACAGTGCCGTCCATGG & hybridization probe \\
\hline KS-p2 & ATTCACGGCGAGGACGGCTCGAAGG & hybridization probe \\
\hline 12J16-ksp1-FW1 & CAAGCGGAATCAATCACA & primer walking \\
\hline 12J16-ksp1-FW2 & TTCTCACTGGCCGACTTT & primer walking \\
\hline 12J16-ksp1-FW3 & GTCGACCAATGCCACAGAAAAC & primer walking \\
\hline 12J16-ksp1-FW4 & AAGATAAAGCAATCTGGCA & primer walking \\
\hline 12J16-ksp1-FW5 & TGTATACCACGAACTTGCC & primer walking \\
\hline 12J16-ksp1-FW6 & GTTGTCGCACACATGAAG & primer walking \\
\hline 12J16-ksp1-FW7 & CCTGCCGCTTTTCCTTATAC & primer walking \\
\hline 12J16-ksp1-RW1 & ATACAAATCCGTGCCCATTC & primer walking \\
\hline 12J16-ksp1-RW2 & TGTTGGAGCATGGGGATGT & primer walking \\
\hline 12J16-ksp1-RW3 & CGTCTATGTCATTGCGCAAGTT & primer walking \\
\hline 12J16-ksp1-RW4 & CGAAGACGAGTAGGGATC & primer walking \\
\hline 12J16-ksp1-RW5 & GATATGCAAGTAGTGTTGG & primer walking \\
\hline KS-63787-f & GCAGATACAGATCCCTACTCGTCTTCG & 5' flanking region cloning \\
\hline KS-63787-HindIII-r & AGAACGAGTGCGCTGTTGGACCCAG & 5' flanking region cloning \\
\hline ACP-63787-XhoI-f & GTAGATGCAAACGGTGTCGATG & 3' flanking region cloning \\
\hline ACP-63787-r & CAGCAGTGCTGAGCCGAAAC & 3' flanking region cloning \\
\hline 2273-f & GAGAGTATGCTGCTCTCGTCATCGC & $\Delta p k s 63787$ screening \\
\hline 2273-r & CCGAGCTGTCGCAGATCGTCG & $\Delta p k s 63787$ screening \\
\hline hph-f & TCTATTCCTTTGCCCTCG & selection marker detection \\
\hline hph-r & GGTTGACGGCAATTTCGATG & selection marker detection \\
\hline
\end{tabular}


Table S5. DPPH free radical scavenging activity of compound 1-7 and fractions of $A$. cinnamomea f101

\begin{tabular}{lll}
\hline Sample & $\mathrm{EC}_{50}(\mu \mathrm{M})$ & ${\text { Scavenging activity }(\%)^{a}}^{a}$ \\
\hline EtOAc layer & $36.99 \pm 0.13^{b}$ & $58.66 \pm 0.36$ \\
$n$-Hexane layer & - & $4.78 \pm 0.53$ \\
$\mathbf{1}$ & $333.10 \pm 0.34$ & $40.86 \pm 0.73$ \\
$\mathbf{2}$ & $40.55 \pm 0.09$ & $93.82 \pm 0.52$ \\
$\mathbf{3}$ & $c$ & - \\
$\mathbf{4}$ & $c$ & - \\
$\mathbf{5}$ & $95.84 \pm 0.34$ & $83.42 \pm 0.10$ \\
$\mathbf{6}$ & $90.45 \pm 0.53$ & $85.56 \pm 0.18$ \\
$\mathbf{7}$ & $\mathrm{c}$ & - \\
BHT & $112.66 \pm 0.64$ & $66.41 \pm 0.83$ \\
\hline
\end{tabular}

${ }^{a}$ Reacted with $20 \mathrm{ppm}$ compounds. ${ }^{b}$ Values represented in ppm. ${ }^{c}$ Values were over $400 \mu \mathrm{M}$. Values shown represented as the mean $\pm \mathrm{SD}(n=4)$. 


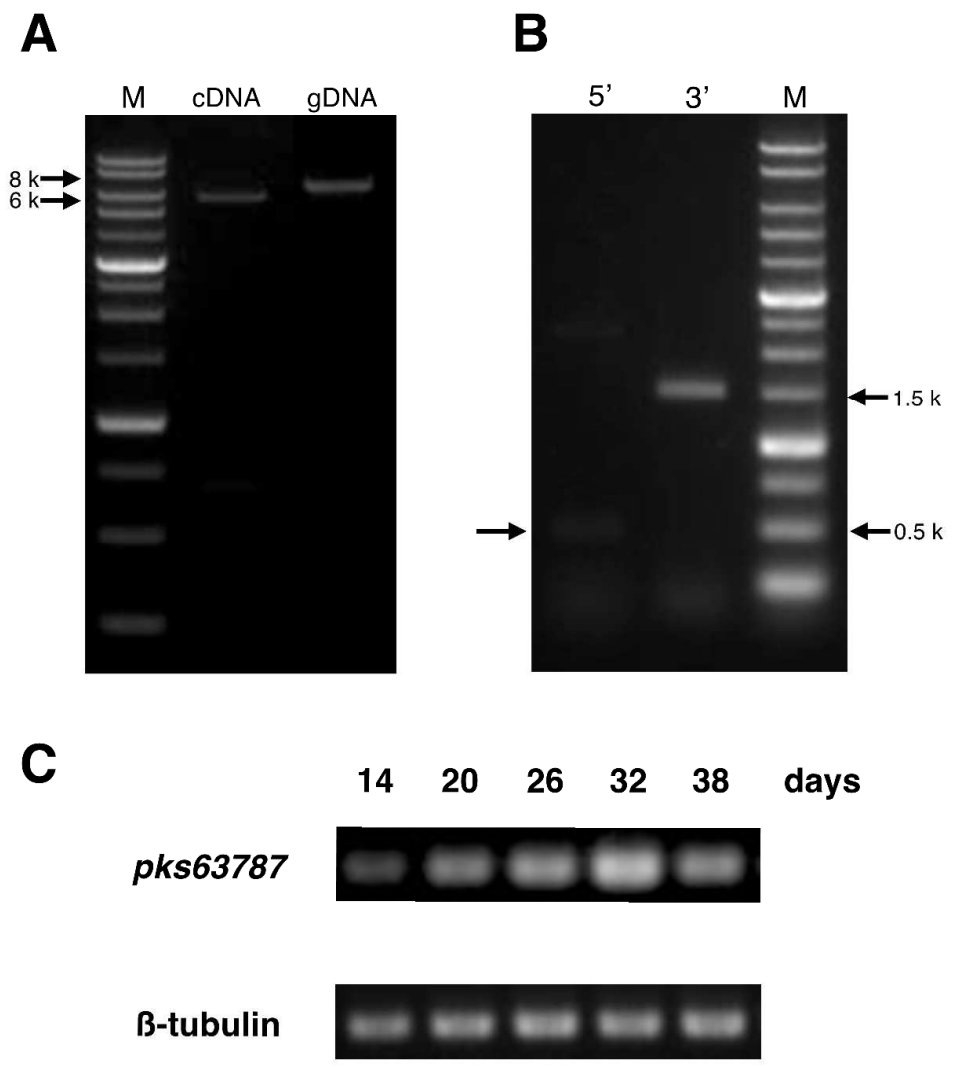

Figure S1. Electrophoresis of RACE and PCR-amplified gDNA, cDNA of pks63787 and expression analysis via RT-PCR. (A) The amplicons of gDNA and cDNA, as the anticipated sizes of 6,977 and 6,531 bp, respectively (arrows). (B) RACE, fragment length of 5' RACE was 518 bp and 3' RACE was 1,532 bp (arrows). M, 1 kb ladder. (C) Gene expression patterns of pks63787 in different culturing period time (days) by RT-PCR. Colonies were grown on PDA and using $\beta$-tubulin gene as an internal control. 


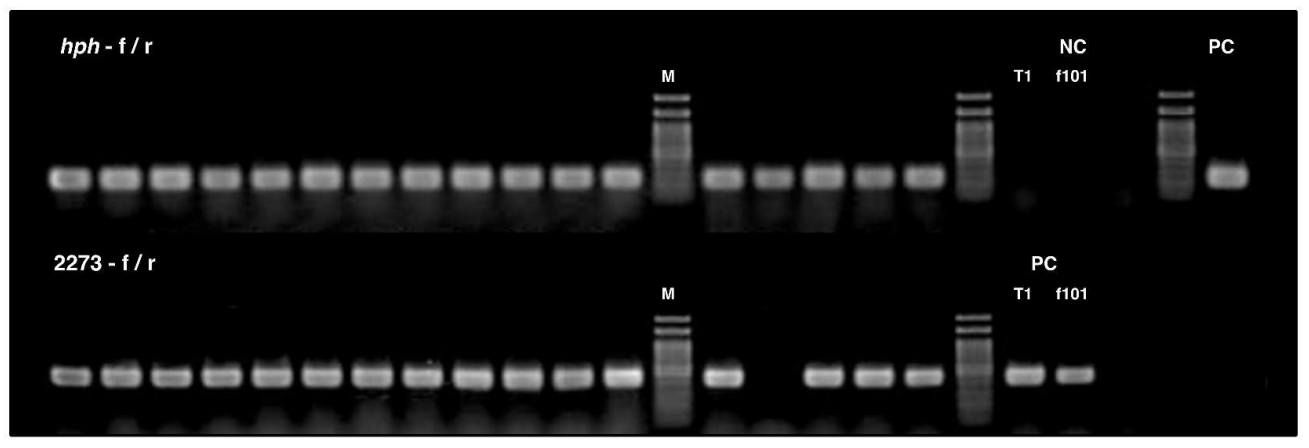

Figure S2. Electrophoresis of PCR amplicon for $\Delta p k s 63787$ screening. $h p h$-f/hph-r primer pair were used for hygromycin cassette detection and 2273-f/2273-r primer pair for the detection of internal region of pks63787; wild type dikaryotic strain T1 and monokaryotic stain f101 as negative control (NC) and positive control (PC) in detection of $h p h$ and pks63787, respectively; M, 100 bp ladder. 